\title{
Review on Mathematical Approaches of Cancer Cell Invasion at Subcellular Level
}

\author{
Nur Azura Noor Azhuan, Sharidan Shafie and Mohd Ariff Admon* \\ Department of Mathematical Sciences, \\ Universiti Teknologi Malaysia 81310 UTM Johor Bahru, Malaysia
}

\begin{abstract}
Metastasis is a process of cancer cell invasion which is the main cause of death among cancer patients. Invasion of a cancer cell on tissue compartment is initiated by the formation of protrusions known as invadopodia and the degradation of extracellular membrane (ECM) barrier. Invadopodia is one of the subcellular views on a cancer cell formed by several processes including ligand formation, signal transduction, chemotaxis, matrix metalloproteinases (MMPs) up regulation and degradation of ECM proteins. Mathematical approach of the cancer cell invasion based on the hypothesis and illustration of previous studies has been considered in order to understand the vivo dynamics of invasion. This study had been done progressively at tissue and cellular levels in the past decades. Recently, mathematical modelling on the study of cancer cell invasion at subcellular level have received much attention since there is potential on anti-invasion therapies for treating the cancer patients. Here, the reviews of mathematical models on cancer cell invasion at subcellular level are able to extend our knowledge on the fields of mathematical medicine and biology.
\end{abstract}

Keywords: invadopodia, mathematical approach, subcellular level

\section{INTRODUCTION}

Cancer is one of the greatest challenges of biomedicine since effective therapies to stop the disease has not yet found. In fact, cancer is recorded as the third leading cause of death in the World Health Organization cancer factsheet (Bernard \& Christopher, 2014). Nonetheless, approaches on understand the biological complexity of cancer including the studies of behaviour of cancer invasion (Aron \& Alissa, 2015) has tremendously increased in the past decades and where nowadays, metastasis is acknowledged as the main cause of the deaths.

There are three natural point of views on describing phenomena of cancer invasion: tissue level, cellular level, and subcellular level. The phenomena that occurs at the tissue level or macroscopic scale typically involves cells with continuum systems, e.g., interactions with external tissues, and phase transition. Meanwhile, at the cellular level or mesoscopic scale refers to the interaction between cells, e.g., epithelial-mesenchymal transition. Finally, at the subcellular level or microscopic scale are the activities taking place within the cell e.g, invadopodia formation.

Mathematical modelling is one of the tools that contributes intensively to the knowledge of cancer invasion, especially to highlight the key components of the invasion. Mathematical modelling on cancer invasion in tissue level began in year 1991. A mathematical model of cancer invasion was pioneered by Chaplain for the diffusion of tumour angiogenesis factor (TAF) into the surrounding tissue (Chaplain \& Stuart, 1991). Later, complex models of cancer invasion have been proposed increasingly in literature with consideration of the continuous approach and system of reaction-chemotaxis-diffusion partial differential equation (PDE). One can refer to Chaplain's group works on continuous improvision of the models of cancer invasion of tissue. Their studies include the effect of

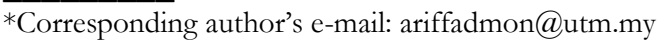


adhesion in cancer cell invasion (Pia et. al., 2014), the effect of over expression of proteolytic enzyme i.euPA and MMPs (Viviet al. 2011) and the role of MMPs and introducing 'matrix suitability' (Niall \& Chaplain, 2013).

On the other hand, in cellular level, Bell (1978) was one of the first contributors on developing a theoretical framework for specific adhesion of cell to cell mediated by the bond in year 1978. These studies had opened new approach on understanding the molecular interaction between cells. Motivated by Bell's theory, Hammer's group developed various models to study the interaction between leukocyte with endothelial cell on the blood vessel wall. Interaction of a cell with ligand-coated surface under flow (Hammer \& Apte, 1992), the effect of cell size (Tees et al. 2002) and the effect of microvillus deformability (Caputo \& Hammer, 2005) are some of the landmark studies at the cellular level.

Recently, development on models of the cancer cell invasion at sub cellular level becomes the main interest based on the biological fact that invadopodia formed initiates the invasion. Hence, the purpose of this review is to highlight the current biological point of view at microscopic scale studies of cancer invasion specifically on invadopodia formation. This review also aims to describe some recent development in mathematical modelling at subcellular point of view to extend the knowledge of cancer invasion.

\section{BIOLOGICAL POINT OF VIEW: KEY COMPONENTS OF INVADOPODIA FORMATION}

Invadopodia is a specialized F-actin rich protrusion localized on the ventral membrane of the cell. As for invadopodia activity, in the intracellular membrane, filamentous (F)-actin organized in bundle to form an actin core structure first. Signalling molecules such as Nck and Rho GTPases cdc42 activate the N-WASP by releasing it, which forms autoinhibitory conformation (Hideki et al. 2005). Next, cofilin nucleated actin polymerization by generating free barbed end at actin filaments causing new filaments to elongate from these barbed ends and maintain the G-actin monomer. Cooperation between Arp2/3 binded to the new actin filaments elongation result in the formation of a branched network of actin (Hadas \& Hava, 2012). Actin cytoskeleton reorganization form prominent ventral protrusion. Epidermal growth factor
(EGF) induced cortactin promotes protease secretion (MMPs) to become de-phosphorylated. Detail of MMPs functions can be found in (Yoshifumi, 2015). Dynamin 2 (Dyn2) is a large GTPase which interacts with cortactin to regulate the actin cytoskeleton (Stanley et. al., 2008). Sequesters cofilin avoid actin depolymerization and cortactin tyrosine phosphorylation of 421 and 466 maturated and stabilized the invadopodia precursor (Christopher et. al., 2011).

CD44 and integrin activates the protease including membrane tethered 1- matrix metalloproteinases (MT1MMP) which is the subgroup type 1 of MMP family. MT1MMP with presence of $\alpha v \beta 3$ integrin then activates MMP2 and MMP9 (Alissa, 2006). Exocyst also plays pivotal role to regulate the secretion of MMPs at focal degradation sites with exocyst component Exo70 or Ser8 (Jianglan, 2009). Cortactin found at the protease secretion to boost the level of secreted protease. ECM is crosslinked with others to form rigid structure barrier, only with help of MT1-MMP can the ECM fibre be degraded.

Adamalysin-like metalloproteinases with thrombospin motifs (ADAMTSs) combine with MMPs also play key roles in ECM proteolytic activation and interaction with tissue inhibitors of metalloproteinases (TIMPs) (Kazuhiro et al. 2015). Actin dynamics act together at the front of the membrane to degrade the ECM fibre (Corinne et. al., 2009). Signalling through $\beta 1$ integrins around actin complex regulates the ability to degrade ECM with combination of proteases at the ventral membrane (Folkman \& Klagsbrun, 1987). Complex structure (combination of actin complex and MMPs) pushes the membrane outward and consequently degrades the ECM fibres and low density lipoprotein (LDL) receptor eliminates the excess active proteinases from tissue and body liquid, and form ligand instead (Kazuhiro et. al., 2015). The ligand binds to extracellular membrane with the help of signalling receptor low receptor-related protein 1 (LRP1) and once again process of actin polymerization occurs with activation from the signalling.

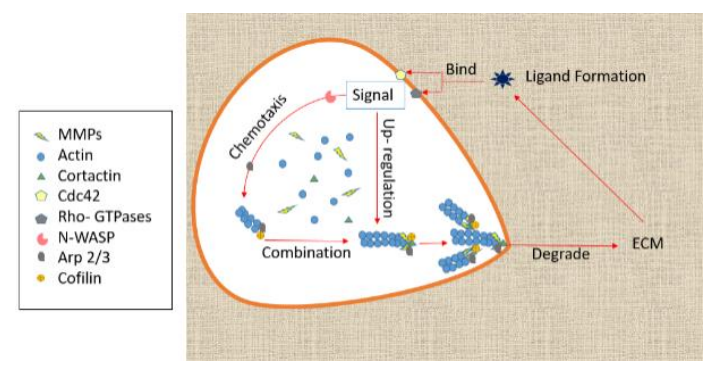

Figure 1. Schematic diagram of invadopodia formation. 
Figure 1 shows the schematic diagram of invadopodia formation. Formation of invadopodia involve loop mechanisms which also includes (i) signalling pathway, (ii) actin polymerization, (iii) activation and movement of matrix metalloproteases (MMPs), (iv) degradation of ECM protein and (v) ligand formation.

\section{MATHEMATICAL MODELING OF INVADOPODIA FORMATION}

Mathematical modelling of cancer invasion at subcellular view are active and limited. This review focused on some recent proposed mathematical model on cancer cell invasion with consideration of the main processes of invadopodia formation.

\section{A. Model of Formation and Maturation of Invadopodia}

Saitou et al. (2012) was first to initiate the derivation of a continuous model based on partial differential equations (PDEs) with fixed boundary to describe the formation and maturation of invadopodia. The model consists of actin polymerization,

$$
n_{t}=\underbrace{d_{n} \Delta n}_{\text {diffusion }}+\underbrace{\nabla \cdot n \nabla \chi(c)}_{\text {separation }}-\underbrace{\gamma_{n} \nabla \cdot n \nabla c_{*}}_{\text {chemotaxis }}
$$

degradation of extracellular membrane, ECM,

$$
c_{t}=-\underbrace{\kappa_{c} c f}_{\text {degradation }} \text {. }
$$

ligand formation,

$$
c_{* t}=\underbrace{d_{c *} \Delta c_{*}}_{\text {diffusion }}+\underbrace{\kappa_{c} c f}_{\text {production }}-\underbrace{\lambda_{c *} c_{*}}_{\text {decay }} .
$$

and movement of matrix metalloprotenases, MMP,

$$
f_{t}=\underbrace{d_{f} \Delta f}_{\text {diffusion }}+\underbrace{\kappa_{f} c_{*}}_{\text {production }}-\underbrace{\lambda_{f} f}_{\text {decay }}-\underbrace{\gamma_{f} \nabla \cdot f \nabla n}_{\text {transportation }} .
$$

Their work is able to generate protrusions with small value of the effect of MMP rate constant. However, the region of actin, $\mathrm{n}>\mathrm{o}$ becomes disconnected as time increases.

\section{B. Model of Signal Transduction}

Corresponding to that problem, Admon proposed an additional variable where he considered signal transduction in the previous model. The signal, $\sigma$ is constructed as follows,

$$
\sigma_{t}=\underbrace{d_{\sigma} \Delta \sigma}_{\text {diffusion }}-\underbrace{\lambda_{\sigma} \sigma}_{\text {decay }} .
$$

Due to the complexity of the model and the signal variable does not depend on other variable, Admon (2015) focused on the studies of the relationship between the presence of signal and the invadopodia formation in one dimensional space. The author treated the boundary as a free boundary using fixed domain method and validated the work with integrated penalty method.

As a result, the boundary moved smoothly and continuously as time progressed demonstrated that the membrane expanded and invadopodia should exist. Admon \& Suzuki (2017) also did a simulation of signal distribution profile to investigate the concentration of the signal. They found out that the highest concentration of the signal is located on the membrane. Admon's work provide a good relation with biological fact. Since the problem involved free boundary, studies in higher dimension is needed to get a clear picture of the relationship of signal transduction with invadopodia formation.

\section{Model of Signal Transduction with Ligand Formation}

Olivier et al. (2017) proposed a model consisting of signal transduction inside the cell described by Laplace equation with Dirichlet condition

$$
\Delta \sigma=0, t \in[0, T], \theta \in \mathbb{T}, \sigma\left|\Gamma_{t}=c^{*}\right| \Gamma_{t},
$$

and ligand formation outside the cell described by Laplace equation with Neumann condition

$$
\Delta c^{*}=0, x \in O_{t}^{e}, c^{*}\left|\omega=0,-\partial_{n} c^{*}\right| \Gamma_{t}=g \mid \Gamma_{t},
$$

in two-dimensional space where $g(t)$ is assumed as the flux of MT1-MMP enzymes inside the cell. They also treated the boundary as a free boundary and first order Cartesian finite difference is proposed to simulate the model. Their result was in good agreement with the theoretical behaviour of invadopodia formation.

Second order Cartesian method proposed in (Olivier \& Poignard, 2017) is a continuation of their studies on the chemical interaction between the cell and its environment. Olivier and Poignard were able to stabilize the ghost fluid method commonly used in free boundary problem. They also focused on improving the velocity extension in numerical simulation. One can follow this improvised velocity extension method to handle the free boundary 
problem model.

\section{Model of Molecules Dynamics of the ECM}

Degradation by MMPS

Minerva (2016) introduced model of an elementary kinetic reaction system representing ECM degradation. The idea was grouping the kinetic reactions based on reaction rate constants. As for the reaction of two different molecules, $A$ and $B$, the mass action law is derived as follows,

$$
\begin{aligned}
& \frac{d[A]}{d t}=-k[A][B]+l[A B], \\
& \frac{d[B]}{d t}=-k[A][B]+l[A B], \\
& \frac{d[A B]}{d t}=k[A][B]-l[A B],
\end{aligned}
$$

where $k$ and $l$ are association and disassociation constant rate, respectively. Two laws were combined which are mass action laws (MALs) and mass conservation laws (MCLs) to handle the nonlinear ODEs. Hence the ODEs were solved explicitly.

Kawasaki et. al., (2017) on the other hand aimed to model the molecules dynamics in cancer cell invasion to the ECM degradation by MT1-MMPs. The model consists of MMP2, TIMP2 and MT1-MMP which denoted as $a, b$ and $c$, respectively. They considered twelve molecules in the complex pathway network (PWN) and denoted their concentration as $X_{i}$ where $i=1,2, \ldots, 12$. One of the concentration ODEs is derived as

$$
\frac{d X_{1}}{d t}=-k_{1} X_{1}\left(X_{2}+X_{5}+X_{8}+2 X_{10}+X_{11}\right)
$$

They followed the method proposed by Minerva (2016) to convert into integrable systems of ODEs.

\section{E. Model of Activation of $M M P 2$}

Activation of MMP2 also play an important role on the degradation of ECM. Hence, several models were proposed to form the activation pathway of MMP2. Hoshino et al. (2012) proposed a model of (MMP2 - TIMP2-MT1-MMP MT1-MMP) complex for MMP2 activation activity. They assumed that limited number of site available for MT1MMP insertation. The derived surface density of MT1-MMP at invadopodia is

$$
M=M_{X}+M_{D}
$$

$$
\begin{gathered}
=\frac{C_{D} k_{X}+\left(k_{X} M_{S}-C_{X}\right) k_{D}}{k_{X} k_{D}} \\
-\frac{k_{X}\left(M_{S}-M_{X} 0\right)-C_{X}}{k_{X}} e^{-k_{X} t} \\
-\frac{\left(C_{D}-k_{D} M_{D 0}\right)}{k_{D}} e^{-k_{D^{t}}}
\end{gathered}
$$

where $M$ is the total amount of MT1-MMP at the region of interest at an invadopodium, which is the sum of the amounts in pool $D\left(M_{D}\right)$ and pool $X\left(M_{X}\right) . C_{D}\left(k_{D}\right)$ and $k_{X}\left(C_{X}\right)$ are insertion rate constant for pools $D$ and $X$, respectively.

$M_{S}$ is the saturated amount of MT1-MMP in pool $D$, and $M_{D 0}$ and $M_{X 0}$ are the amounts of unbleached MT1-MMP in pools $\mathrm{X}$ and $\mathrm{D}$, respectively.

Most recently, Itano (2018) studies the biochemical reaction of activation of MMP by introducing the matrix metalloproteinase $2(\mathrm{MMP} 2)$ activation model. The author generalized the homodimer pathway network which consists of 3monomer and formed $\mathrm{N}$ monomer pathway network. Mass conservation law is applied into themodel, the $N(N+1)$ molecules ODEs is simplified to $N$ group ODEs. Each complex ODE is constructed as

$$
\frac{d X_{l, m}(t)}{d t}=-A_{l, m}(t) X_{l, m}(t)+f_{l, m}(t)
$$

where $A_{l, m}(t)$ and $f_{l, m}(t)$ are variables with reaction group variable, $\varsigma_{m}(t)$ and reaction rate

constants $k_{m}$ and $l_{m}(m=1, \ldots, N)$. The ODEs are solved explicitly.

\section{CONCLUSION}

As a summary, models corresponding to the formation of invadopodia include the formation and maturation of invadopodia, signal transduction with or without ligand formation, ECM degradation and activation of MMP2 were proposed and analysed intensively. Model proposed by Saitou et al. (2012) which consists of actin polymerization, degradation of ECM, ligand formation and activation and movement of ECM is a good start in modelling invadopodia. Model proposed by Admon (2015) provided a good relation between the presence of signal with invadopodia formation. Olivier et. al., (2017) were 
able to successfully simulate the signal transduction with ligand formation and shows super convergent in two level. Kawasaki et. al., (2017) proposed new method to integrate nonlinear ODEs to ODEs to solve the system explicitly. While Itano (2018) proposed a model of $\mathrm{N}$ monomer for the activation pathways of MMP2.

Studies on cancer cell invasion at subcellular view had been done currently and actively. However, there are a lot of improvements that can be proposed in the future i.e signal transduction simulation in twodimension, ECM degradation corresponding to the ligand formation and combination of complex MMPs with actins. This study should be continued in order to model the complete mechanism of invadopodia formation mathematically and able to contribute knowledge in the field of mathematical medicine and biology.

\section{ACKNOWLEDGEMENT}

The authors would like to thank MyBrainSC and FRGS grant (5Fo98) for the financial supports. 


\section{REFERENCES}

Admon, M. A. \& Suzuki, T. 2017, Signal transduction in the invadopodia formation using fixed domain method. Journal of Physics: Conference series, 890.

Alissa, M. W. 2006, Invadopodia: Specialized Cell Structures for Cancer Invasion. Clinical and Experimental Metastasis, 23, pp. 97-105.

Aron, P. \& Alissa, M. W. 2015, Regulation of Invadopodia by Mechanical Signalling. Experimental Cell Research, 343, pp. 89-95.

Bell, G. I. 1978, Models for the specific adhesion of cells to cells. Science, 200, 618.

Bernard, W. S. \& Christopher, P. W. 2014, World Cancer Report 2014. France. World Health Organization.

Caputo, K. E.\& Hammer, D. A. 2005, Effect of Microvillus Deformability on Leukocyte Adhesion Explored Using Adhesive Dynamics Simulations. Biophysical Journal, 89,187200 .

Chaplain, M. A. J. \& Stuart, A. M. 1991, A Mathematical Model for the Diffusion of Tumour Angiogenesis Factor into the Surrounding Host Tissue. Journal of Mathematics Applied in Medicine and Biology, 8, pp. 191-220.

Christopher, C. M., Matthew, O., Marco, A. O. M., Jose, J. B. C., John, C., Anthony, J. K. \& Hava, G. H. 2011, An EGFR-Src-Arg- Cortactin Pathway Mediates Functional Maturation of Invadopodia and Breast Cancer Cell Invasion. Cancer Research, 71(5), pp. 1730-1741.

Corinne, A. R., Olivier, D., Bertrand, F., Emmanuelle, P. \& Marc, R. B. 2009, Actin Mechinery and Mechanosensitivity in Invadopodia, Podosome and Focal Adhesions. Journal of Cell Science, 122(17), pp. 3037-3049.

Dhisa Minerva. 2016, Mathematical Studies on ECM Degradation and Angiogenesis. PHD Thesis, Osaka University, Japan.

Hadas, S. B. \& Hava, G. H. 2012, Invadopodia: The Leading Force. European Journal of Cell Biology, 91, pp. 896901.

Folkman, J. \& Klagsbrun, M. 1987, Angiogenic factors. Science, 235 (4787), 442447.
Hammer, D. A. \& Apte, S. M. 1992, Simulation of cell rolling and adhesion on surfaces in shear flow: general results and analysis of selectin-mediated neutrophil adhesion. Biophysical Journal, 63, pp. 35-57.

Hideki, Y., Mike, L., Stephan, K., Corina, S., Salvatore, C., Marc, S., Jeffrey, S., Robert, E., Hiroaki, M., Tadaomi, T. \& John, C. 2005, Molecular Mechanism of Invadopodium Formation: The Role of the N-WASPArp2/3 Complex Pathway and Cofilin. The Journal of Cell Biology, 163(3), pp. 441-452.

Hoshino, D., Koshikawa, N., Suzuki, T., Ichikawa, K., Quaranta, V., Weaver, A. \&Seiki, M. 2012, Establishment and validation of computational model for MT1-MMP dependent ECM degradation and intervention strategies. PLoS Computational Biology, e1002479.

Itano, K. 2018, Mathematical modeling and analysis of the pathway network consisting of symmetrical complexes with $\mathrm{N}$ monomers, like the activation of MMP2. Quantitative Biology, arXiv:1803.01997.

Jianglan, L., Peng, Y., Vira, V. A., Susette, C. M. \& Wei, G. 2009, The Role of the Exocystin Matrix Metalloproteinase Secretion and Actin Dynamics during Tumor Cell Invadopodia Formation. Molecular Biology of the Cell, 20, pp. 3763-3771.

Kawasaki, S., Minerva, D., Itano, K. \& Suzuki, T. 2017, Finding Solvable Units of Variables in Nonlinear ODEs of ECM Degradation Pathway Network. Computational and Mathematical Method in Medicine, 5924270.

Kazuhiro, Y., Gillian, M. \& Linda, T. 2015, Extracellular Regulation of Metalloproteinases. Matrix Biology, 44(46), pp. 255-263.

Mohd Ariff Bin Admon. 2015, Mathematical Modeling and Simulation in an Individual Cancer Cell Associated with Invadopodia Formation. PHD Thesis, Osaka University, Japan.

Niall, E. D. \&Chaplain, M. A. J. 2013, Mathematical Modelling of Cancer Invasion: The Role of Membrane Bound Matrix Metalloproteinases. Frontiers in Oncology, 70 (3), pp. 1-9. 
Olivier, G., Masahito, O., Poignard, C. \& Takashi, S. 2017, Free boundary problem for cell formations: theoretical and numerical aspects. Mathematical Biology, 75, pp. 263-307.

Olivier, G. \&Poignard, C. 2017, Super convergent second order Cartesian method for solving free boundary problem for invadopodia formation. Journal of Computational Physics, 339, pp. 412-431.

Pia, D., Dumitru, T., Alf, G. \& Chaplain, M. A. J. 2014, Mathematical Modelling of Cancer Invasion: Implications of Cell Adhesion Variability for Tumour Infiltrative Growth Patterns. Journal of Theoretical Biology, 361, pp. 41-60.

Saitou, T., Rouzimaimaiti, M., Koshikawa, N., Seiki, M., Ichikawa, K. \& Suzuki, T. 2012, Mathematical Modeling of Invadopodia Formation. Journal of Theoretical Biology, 298, pp. 138-146.

Stanley, S. S. Andrew, H. K. \& Peter, L. 2008, Invadopodia: At the Cutting Edge of Tumour Invasion. Journal of Clinical Neuroscience, 15, pp. 725-737.

Tees, D. F.J., Chang, K. C., Stephen, D. R. \& Hammer, D. A. 2002, Simulation of Cell Adhesion to Bioreactive Surfaces in Shear: The Effect of Cell Size. Industrial and Engineering Chemistry Research, 41 (3), pp. 486493.

Vivi, A., Alf, G., Georgios, L., Andrew, P. S. \& Chaplain, M. A. J. 2011, Mathematical modeling of cancer cell invasion of tissue: biological insight from mathematical analysis and computational simulation. Mathematical Biology, 63, pp. 141-171.

Yoshifumi, I. 2015, Membrane-Type Matrix Metalloproteinases: Their Functions and Regulations. Matrix Biology, 44(46), pp. 207-223. 